\title{
Evaluation of Anticorrosion Performance for Composite Coatings with Polyaniline and Core-Shell Fluorine-containing Polyacrylate Emulsion
}

\author{
Yufeng $\mathrm{Li}^{1, \text { a }}$, Xiaohui Gao ${ }^{2, \mathrm{~b}}$, Jingjing Zhu ${ }^{1, \mathrm{c}}$ and Di Wang ${ }^{1, \mathrm{~d}}$ \\ ${ }^{1}$ College of Material Science and Engineering, Qiqihar University, Qiqihar 161006, China \\ ${ }^{2}$ College of Chemistry and Chemical Engineering, Qiqihar University, Qiqihar 161006, China \\ alyf1170@163.com, boxh1172@163.com, czhujingjing.hy@163.com, dwang_di111@126.com
}

Keywords: Polyaniline, polyacrylate, fluorine, emulsion, anticorrosion.

\begin{abstract}
Composite emulsion coating with polyaniline and core-shell fluorine-containing polyacrylate emulsion (PANI/ FPAc) was prepared for protecting Q235 steel in this paper. The structure of PANI emulsion particles and core-shell FPAc emulsion particles was observed by transmission electron microscope (TEM). The hydrophobic properties of the composite emulsion coatings were tested by contact angle tester. Electrochemical methods such as electrochemical impedance spectroscopy (EIS) and Tafel curve were used to evaluate the anticorrosion performance of the composite emulsion coatings. The results showed that PANI/FPAc composite emulsion coating had better anticorrosion properties when its proportion reached 1:1.
\end{abstract}

\section{Introduction}

PANI is a kind of conducting polymers which has relatively low cost, good environmental stability, electrical conductivity, and corrosion resistance [1]. It is used in anticorrosion coatings as a corrosion inhibitor for metals by mixing with organic polymers [2, 3]. In recent years, acrylic (acrylate) resin as binder for PANI was researched widely due to its extraordinary properties such as high adornment properties and good film formation [4]. But for these reports, acrylic (acrylate) resin was oil-soluble which need to dilute with organic solvent. Water-borne anticorrosion coatings have become a new trend in the coatings industry with the scientific development and environmental protection request [5]. However, the shortcomings of the water-borne polyacrylate emulsion, such as poor resistance to water, weak adhesion, as well as inadequate corrosion resistance limit their application in anticorrosion coatings. In addition, PANI powder is difficult to disperse in waterborne polyacrylate emulsion, which affects the corrosion resistance performance of the coating. Emulsion polymerization pathway is recognized as one of the ways to improve the process ability of PANI [6]. The authors prepared composite emulsion coatings with PANI emulsion and fluorocarbon acrylate emulsion [7]. In this paper, anticorrosion performance of obtained PANI/core-shell FPAc composite emulsion coatings was evaluated by EIS and Tafel curves.

\section{Experiments}

Preparation of Core-Shell FPAc Emulsion. The $\mathrm{pH}$ buffer agent, one third of emulsifier, initiator, and a half of core pre-emulsion(containing MMA, BA, MAA, one third of emulsifier) were added into a four-neck flask with a thermometer, reflux condenser and a mechanical stirrer. Dropped the other core pre-emulsion and one third of the initiator into the flask when the temperature reached $80^{\circ} \mathrm{C}$. After $40 \mathrm{~min}$, added the shell pre-emulsion (containing MMA, BA, MAA, DFHM, one third of emulsifier) and the remained one third of initiator to the flask. Heated up the temperature to $85^{\circ} \mathrm{C}$, and maintain this temperature for 1 hour.

Preparation of PANI Emulsion. Predetermined amount of DBSA was dissolved in deionized water into a $250 \mathrm{ml}$ three-neck flask, the monomer of aniline was introduced slowly with vigorous mechanical stirred and the stir continued for $1 \mathrm{~h}$ to make pre-emulsion. Subsequently, the APS 
solution, at the stoichiometric ratio, was dropped into the reactor to initiate the polymerization. The reaction lasted for $6 \mathrm{~h}$ at $5^{\circ} \mathrm{C}$.

Preparation of PANI/Core-Shell FPAc Coating. The composite emulsion was produced by mixing PANI emulsion and core-shell FPAc emulsion. The composite emulsion coatings were prepared by brushing the composite emulsion onto Q235 steel. The coated Q235 steel was dried in an oven at $30^{\circ} \mathrm{C}$ for $24 \mathrm{~h}$. Composite emulsion coatings of PANI/PAc and PANI/FPAc were prepared with the same method for comparing.

Characterization and Testing. The structure of core-shell FPAc and PANI particles was observed at TEM (Model H-7650). The contact angle of PANI/PAc, PANI/FPAc and PANI/coreshell FPAc composite coatings was texted by a contact angle tester (Model JY-82). The anticorrosion properties of composite coatings were evaluation through electrochemical experiments in $3.5 \mathrm{wt} \% \mathrm{NaCl}$ aqueous solutions with the samples as working electrode, a platinum electrode as counter electrodes and an $\mathrm{Ag} / \mathrm{Ag}^{+}$electrode as reference electrodes on an electrochemical analyzer(Model LK2005).

\section{Results and Discussion}

TEM photos of PANI and core-shell FPAc emulsion particles were shown in Fig.1A and Fig.1B, respectively. It can be found that PANI emulsion particles have nanometer fiber-like structure with about 200nm of the diameter, and FPAc emulsion particles have a core-shell structure with about $70 \mathrm{~nm}$ of the core diameter and $15 \mathrm{~nm}$ of the shell thickness.
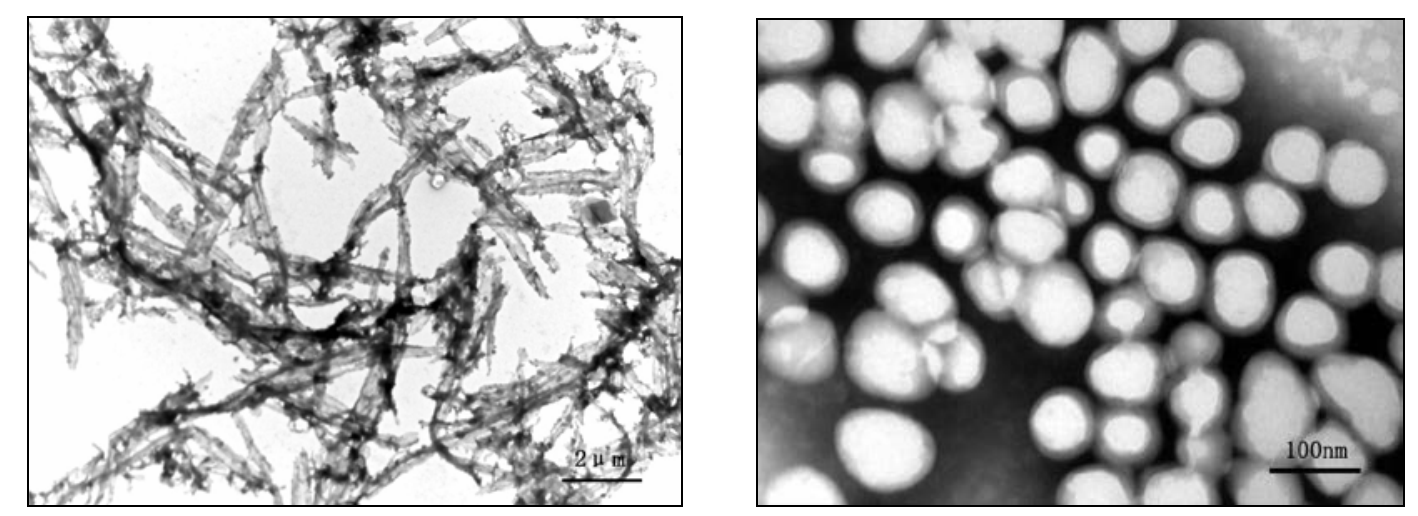

Fig. 1 TEM photos of PANI and core-shell FPAc emulsion particles

Fig. 2 shows the water contact angle of PANI/PAc emulsion coating is $65.06^{\circ}$, PANI/FPAc emulsion coating is $77.71^{\circ}$ and PANI/core-shell FPAc emulsion coating is $88.66^{\circ}$. These results showed that the core-shell PANI/FPAc emulsion coating is more hydrophobic than others. This because that the core-shell structure is more conducive to fluorine segment migrates to the surface. Good hydrophobic properties could improve the corrosion resistance of the composite coatings.
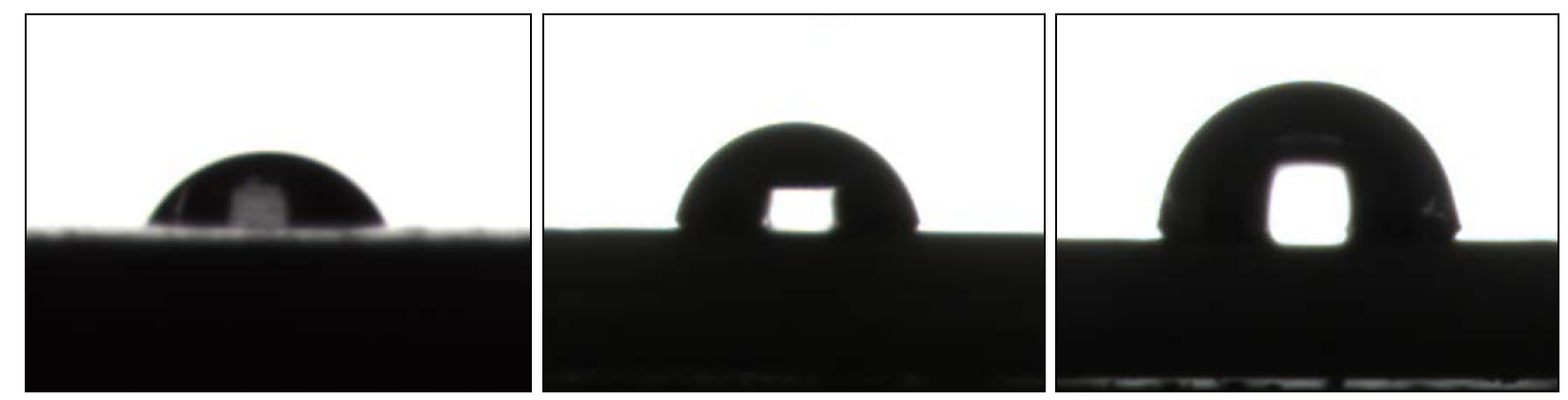

Fig.2 Water contact angle of PANI/PAc, PANI/FPAc and PANI/core-shell FPAc emulsion coatings 
Tafel curve and EIS of PANI/PAc, PANI/FPAc, and PANI/core-shell FPAc composite emulsion coatings were shown in Fig.3 and Fig.4. The PANI/core-shell FPAc composite emulsion coating has a lower corrosion current density and higher impedance than PANI/PAc and PANI/FPAc composite emulsion coatings. This suggests that the PANI/core-shell FPAc composite coating had better performance against corrosion.

In further, properties of PANI/core-shell FPAc composite emulsion coatings with different proportion were studied. The water contact angle of PANI/core-shell FPAc coatings with different proportion was given in Fig.5. The water contact angle decreases slowly with the PANI emulsion increases at first, and decreases sharply when the PANI/core-shell FPAc ratio exceeds 1:1.

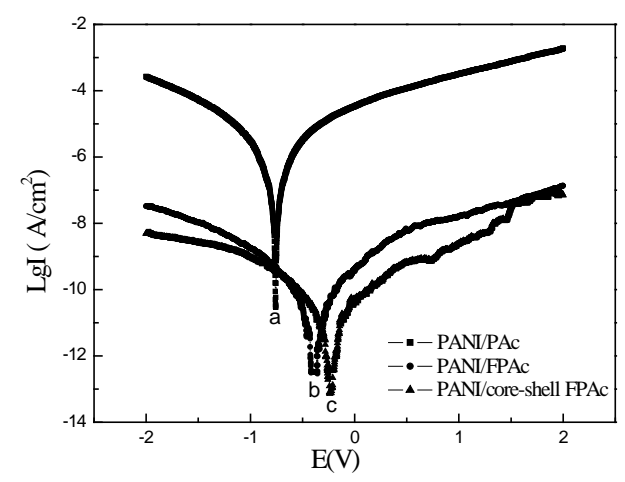

Fig.3 Tafel curve of composite emulsion coatings

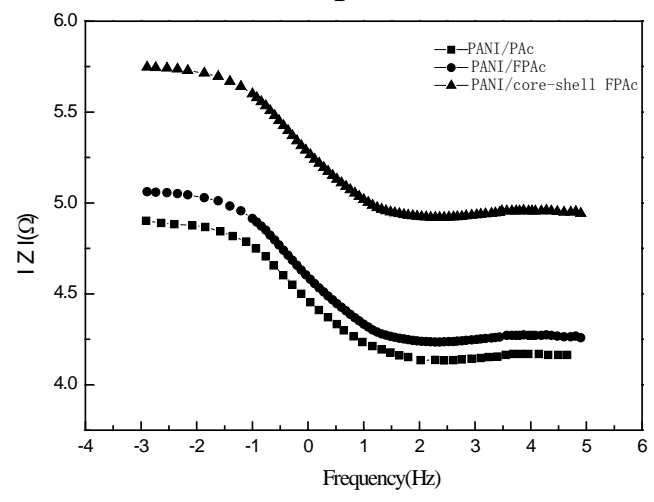

Fig.4 EIS of composite emulsion coatings

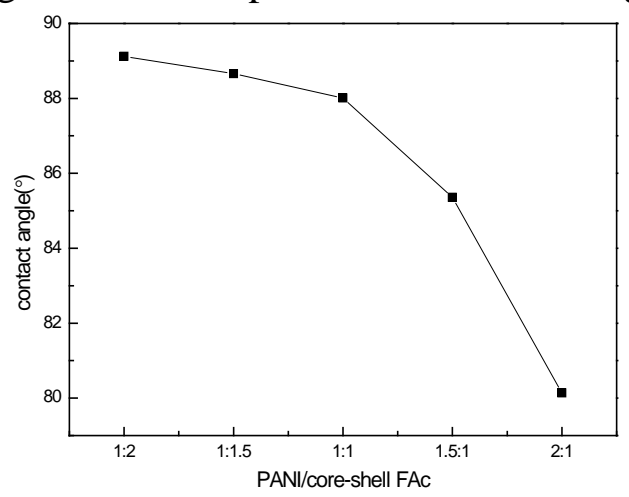

Fig.5 Water contact angle of PANI/core-shell FPAc coatings with different proportion

The anticorrosion performance of PANI/core-shell FPAc coatings with different proportion was further tested by Tafel curve and EIS. The results were provided in Fig.6 and Fig.7. The corrosion current density decreases first and then increases with the proportion of PANI and core-shell FPAc increases. The impedance increases first and then decreases with the proportion of PANI and core-shell FPAc increases. This can be understood that the passivity ability of composite coating was enhancement when the PANI content increases in the film. But too much PANI content makes the hydrophobic performance decrease. The composite coating has best anticorrosion properties when the proportion of PANI and core-shell FPAc was 1:1. 


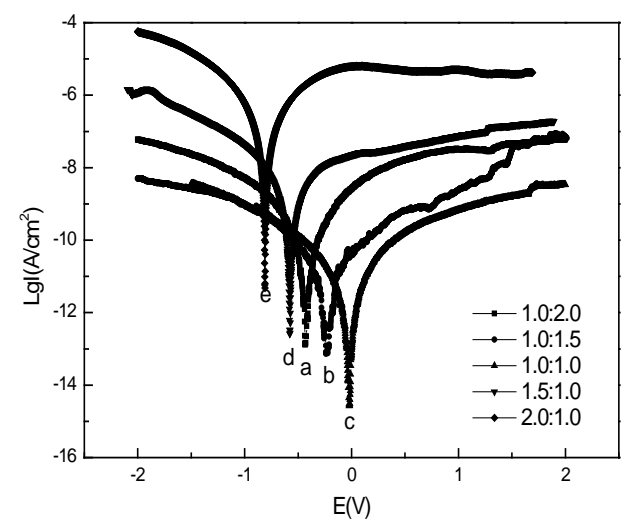

Fig.6 Tafel curve of composite emulsion coatings with different PANI:core-shell FPAc

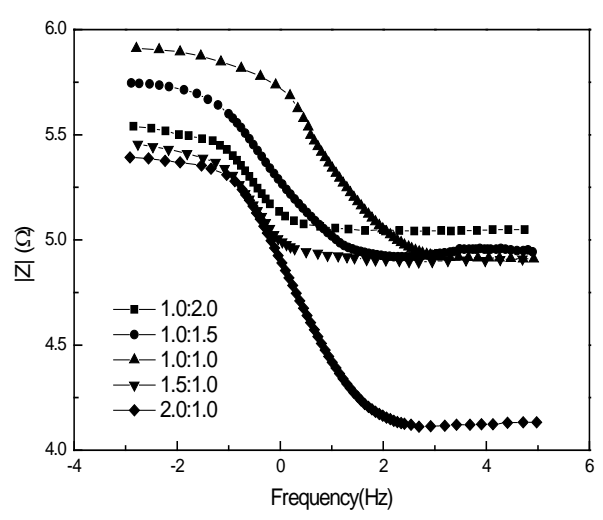

Fig.7 EIS of composite emulsion coatings with different PANI:core-shell FPAc

\section{Summary}

PANI emulsion and core-shell FPAc emulsion were synthesized, and then mixed the two emulsions to prepare the composite coating for anticorrosion of Q235 steel. TEM photos showed the PANI emulsion particles have filler-like structure and the FPAc emulsion has a core-shell structure. Contact angle test results showed that the PANI/core-shell FPAc composite coating has better hydrophobic performance than PANI/PAc and PANI/FPAc. The composite emulsion coating showed best anticorrosion properties when the proportion of PANI and core-shell FPAc was 1:1, the impedance reached $10^{5.7} \Omega$, and the corrosion current density was only $10^{-11} \mathrm{~A} / \mathrm{cm}^{2}$.

\section{Acknowledgements}

The authors are grateful for the financial support of the Natural Science Foundation of Heilongjiang Province of China (No. E201138).

\section{References}

[1] A. Pud, N. Ogurtsov, A. Korzhenko, et al, Some aspects of preparation methods and properties of polyaniline blends and composites with organic polymers, Prog. Polym. Sci. 28 (2003) 1701-1753.

[2] A. Mostafaei, F. Nasirpouri, Epoxy/polyaniline-ZnO nanorods hybrid nanocomposite coatings: Synthesis, characterization and corrosion protection performance of conducting paints, Prog. Org. Coat. 77 (2014) 146-159.

[3] M. Sababi, J.S. Pan, P.E. Augustsson, et al, Influence of polyaniline and ceria nanoparticle additives on corrosion protection of a UV-cure coating on carbon steel, Corros. Sci. 84 (2014) 189-197.

[4] S. Jafarzadeh, M. Johansson, P. Sundell, et al, UV-curable acrylate-based nanocomposites: effect of polyaniline additives on the curing performance, Polym. Adv. Technol. 24 (2013) 668-678.

[5] T.Gurunathan, C.R.K. Rao, R. Narayan, et al, Synthesis, characterization and corrosion evaluation on new cationomeric polyurethane water dispersions and their polyaniline composites, Prog. Org. Coat. 76 (2013) 639-647.

[6] S. Palaniappan, A. John, Polyaniline materials by emulsion polymerization pathway, Prog. Polym. Sci. 33 (2008) 732-758.

[7] Y.F. Li, Z.P. Zeng, D. Wang, et al, Polyaniline/Fluorocarbon Composite Emulsion Coatings for Anticorrosion to Mild Steel, Appl. Mech. Mater. 151 (2012) 323-326. 